\title{
Upregulation of thyroid hormone receptor interactor 13 is associated with human hepatocellular carcinoma
}

\author{
LINLING JU $^{1 *}$, XUEFENG LI ${ }^{2 *}$, JIANGUO SHAO ${ }^{1}$, RUJIAN LU ${ }^{1}$, \\ YIFAN WANG $^{1}$ and ZHAOLIAN BIAN ${ }^{1}$ \\ ${ }^{1}$ Nantong Institute of Liver Disease, Nantong Third People's Hospital, Nantong University, Nantong, Jiangsu 226006; \\ ${ }^{2}$ Department of Medical Oncology, The First Affiliated Hospital of the University of South China, \\ Hengyang, Hunan 421001, P.R. China
}

Received March 7, 2018; Accepted September 18, 2018

DOI: $10.3892 /$ or.2018.6767

\begin{abstract}
Hepatocellular carcinoma (HCC) is the second leading cause of cancer-related mortality worldwide. Thyroid hormone receptor interactor 13 (TRIP13) has been reported to promote nonhomologous end joining (NHEJ). However, the role of TRIP13 in the molecular pathogenesis of HCC remains to be elucidated. The aim of the present study was to investigate the role and potential mechanism of TRIP13 in HCC. Real-time PCR and western blotting were used to detect the expression of TRIP13 in 47 paired HCC and adjacent liver tissues. The Cancer Genome Atlas (TCGA) database was used to collect TRIP13 expression data in HCC. Loss-of-function siRNA assays were performed to alter TRIP13 expression. Cell proliferation, migration and invasion capabilities were investigated using CCK-8, wound healing and Transwell assays, while cell cycle distribution and apoptosis were assessed using flow cytometry. The expression of TRIP13 was upregulated in HCC tissues and cell lines. We analyzed the association between TRIP13 expression and patient prognosis. Patients with higher TRIP13 expression had significantly shorter survival periods compared with patients with lower TRIP13 expression. CCK-8, wound healing and Transwell assays revealed that TRIP13 downregulation inhibited the proliferation, migration and invasion of HCC cells. TRIP13 downregulation resulted in increased apoptosis and cell cycle arrest at S-phase. Finally, we found that loss of TRIP13 reduced the expression of cellular NHEJ proteins such as KU70, KU80 and PRKDC, and these results suggest that loss
\end{abstract}

Correspondence to: Dr Zhaolian Bian and Dr Yifan Wang, Nantong Institute of Liver Disease, Nantong Third People's Hospital, Nantong University, 60 Middle Qingnian Road, Nantong, Jiangsu 226006, P.R. China

E-mail: bianzhaolian1998@163.com

E-mail:wangyifan1988@hotmail.com

*Contributed equally

Key words: hepatocellular carcinoma, TRIP13, proliferation, apoptosis, cell cycle of TRIP 13 impairs the NHEJ repair process in HCC cells. Collectively, these results provide evidence that TRIP13 may act as a tumor promoter during HCC development and could serve as a potential therapeutic target for this disease.

\section{Introduction}

Hepatocellular carcinoma (HCC) is one of the most prevalent malignant diseases and the second leading cause of cancerrelated deaths worldwide $(1,2)$. HCC is characterized by a high probability of recurrence and metastasis. In areas such as China, where there is a high incidence of hepatitis $\mathrm{B}$, the problem is exacerbated $(3,4)$. Only $10-15 \%$ of HCC patients are suitable candidates for curative treatments, such as surgical resection and liver transplantation (5-7). The underlying molecular mechanisms of HCC need to be further elucidated in order to develop novel prognostic indicators and targeted therapies for improved clinical management.

Thyroid hormone receptor interacting protein 13 (TRIP13, also known as HPV16E1BP) is a member of the functionally diverse AAA+ ATPase family of proteins and contributes to homologue pairing, synapsis and recombination during meiosis (8-10). TRIP13 is located in the nucleus and is a novel kinetochore protein that interacts directly with mitotic checkpoints (11). TRIP13 was first identified in yeast twohybrid screening as a protein fragment that was associated with thyroid hormone receptors in a hormone-independent fashion $(12,13)$. TRIP13 is the mouse orthologue of pachytene checkpoint 2 (Pch2), a checkpoint for synapsis before doublestrand break (DSB) repair and recombination in yeast and Caenorhabditis elegans $(8,14)$. Human TRIP13 has also been identified as an oncogene (9). TRIP13 is overexpressed in a number of human cancers $(12,15)$; for example, in squamous cell carcinoma of the head and neck (SCCHN), TRIP13 overexpression leads to aggressive, treatment-resistant tumors and enhanced repair of DNA damage (8). Recent studies have revealed that TRIP13 promotes nonhomologous end-joining (NHEJ) and its overexpression results in cellular transformation and resistance to chemotherapeutic agents, which suggests that aberrant expression of TRIP13 may be associated with tumor progression (8). However, little is known concerning the roles or direct mechanisms of TRIP13 in HCC. The aim of 
the present study, therefore, was to elucidate the function and mechanism of TRIP13 in HCC.

The results of the present study revealed that the expression of TRIP13 was elevated in HCC. Furthermore, TRIP3 expression was correlated with cancer progression and malignancy. We investigated the effect of TRIP13 downregulation on the proliferation, invasion, cell cycle progression and apoptosis of HCC cell lines in vitro. These results may be useful for the development of novel prognostic markers and therapeutic targets for cancer.

\section{Materials and methods}

Cell lines and clinical samples. HCC cell lines Huh-7, SNU-423 and the normal human liver LO2 cell line were purchased from the Chinese Academy of Sciences (Shanghai, China). All cells were cultured in Dulbecco's modified Eagle's medium (DMEM) (Gibco; Thermo Fisher Scientific, Inc., Waltham, MA, USA) supplemented with $10 \%$ fetal bovine serum (FBS) at $37^{\circ} \mathrm{C}$ with $5 \% \mathrm{CO}_{2}$. A total of 47 paired $\mathrm{HCC}$ samples from primary tumor and adjacent non-tumor sites were obtained from patients with primary liver cancer prior to any therapy. The adjacent non-tumor area was subsequently verified to be free of tumor infiltration using histology. All samples were obtained from patients at Nantong Third People's Hospital Affiliated to Nantong University between September 2015 and July 2016. The pathological stage of HCC was determined according to the International Union Against Cancer (UICC) Tumor-Node-Metastasis (TNM) Classification. The characteristics of the participants in this study are summarized in Table I. The study was approved by the Ethics Committee of Jiangsu Province Medical Association and written informed consent was obtained from each participant. All samples were frozen immediately after collection and stored at $-80^{\circ} \mathrm{C}$ until further use.

Quantitative real-time-PCR ( $q R T-P C R)$. Total RNA was purified from the HCC tissue and cultured cells using TRIzol (Thermo Fisher Scientific, Inc.), following which RNA was reverse transcribed using a PrimeScript ${ }^{\mathrm{TM}}$ RT Reagent kit (Takara Biotechnology Co., Ltd., Dalian, China) according to the manufacturer's protocol. PCR reactions were performed using the SYBR Green Master Mix (Vazyme, Piscataway, NJ, USA). The primers used were as follows: TRIP13, forward, 5'-GCGTGGTCAATGCTGTCTTG-3' and reverse, 5'-CACGTCGATCTTCTCGGTGA-3'; $\beta$-actin, forward, 5'-CGACAGGATGCAGAAGGAGA-3' and reverse, 5'-CATCTGCTGGAAGGTGGACA-3' (Sangon Biotech Co., Ltd., Shanghai, China). Thermocycling conditions were as follows: $95^{\circ} \mathrm{C}$ for 5 min followed by 40 cycles of a 10 -sec denaturation at $95^{\circ} \mathrm{C}$ and 30 -sec annealing at $60^{\circ} \mathrm{C}$. Experiments were performed in triplicate and fold amplification was calculated using the $2^{-\Delta \Delta \mathrm{Cq}}$ method (16).

Immunohistochemistry (IHC). Tissues were formalin-fixed, paraffin-embedded and sectioned $(6 \mu \mathrm{m})$. For immunohistochemical staining, sections were deparaffinized and incubated with $3 \% \mathrm{H}_{2} \mathrm{O}_{2}$ for $15 \mathrm{~min}$. Antigen retrieval was performed by boiling the sections in citrate buffer ( $\mathrm{pH}$ 6.0) in a microwave for $10 \mathrm{~min}$. After cooling, the sections were incubated with
Table I. Clinical characteristics of the HCC cases used in this study.

\begin{tabular}{lc}
\hline Clinical variables & Data \\
\hline Number of patients & 47 \\
Mean age \pm SD (years) & $56 \pm 11$ \\
Sex, n (\%) & \\
Female & $18(38.30)$ \\
Male & $29(61.70)$ \\
Serum AFP (ng/ml), n (\%) & \\
$\leq 400$ & $36(76.60)$ \\
$>400$ & $11(23.40)$ \\
HBV infection, $\mathrm{n}(\%)$ & \\
Positive & $38(80.9)$ \\
Negative & $9(19.1)$ \\
Largest tumor diameter, & $5.7 \pm 3.4$ \\
mean \pm SD (cm) & \\
TNM stage (I/II/III), n (\%) & \\
I & $2(4.25)$ \\
II & $30(63.83)$ \\
III & $15(31.92)$ \\
Lymph node metastasis, $\mathrm{n}(\%)$ & $20(42.55)$ \\
Positive & $27(57.45)$ \\
Negative & \\
\hline
\end{tabular}

HCC, hepatocellular carcinoma; AFP, $\alpha$-fetoprotein; HBV, hepatitis B virus; TNM, tumor, node, metastasis.

$10 \%$ non-immune goat serum for $30 \mathrm{~min}$. Specimens were subsequently incubated with antibodies overnight at $4^{\circ} \mathrm{C}$. After rinsing with PBS, the sections were incubated with a horseradish peroxidase (HRP)-conjugated secondary antibody for $30 \mathrm{~min}$ at room temperature. PBS was then used to rinse the specimens, after which 3,3'-diaminobenzidine (DAB; Maixin-Bio, Guangzhou, China) was applied for $60 \mathrm{sec}$. Hematoxylin was applied for nuclear staining and the stained specimen was cover-slipped for imaging by light microscopy (Olympus Corp., Tokyo, Japan).

Cell transfection and transduction. Huh-7 cells were seeded in 6 -well plates in medium containing $10 \%$ serum to allow cells to reach $50-60 \%$ confluence on the day of transfection. Cells were transfected with $10 \mathrm{nM}$ siRNA targeting TRIP13 or a nontargeting sequence control siRNA using Lipofectamine 2000 (Invitrogen; Thermo Fisher Scientific, Inc.) according to the manufacturer's protocol. Gene expression was analyzed by RT-PCR $48 \mathrm{~h}$ following transfection. TRIP13 was downregulated by TRIP13 siRNA (Shanghai GenePharma Co., Ltd., Shanghai, China). The TRIP13 siRNA oligonucleotide sequence was as follows: GACCAGAAAUGUGCAGUCU. For TRIP13 overexpression, Huh-7 cells were transfected with GV141-Flag-TRIP13 or GV141-Flag control vector (Sangon Biotech Co., Ltd.). Finally, qRT-PCR and western blotting were performed to validate the transfection efficacy. All assays were performed in triplicate. 
Western blotting. Frozen tissues were lysed in radioimmunoprecipitation lysis buffer, including phosphatase inhibitor cocktail (Beyotime Institute of Biotechnology, Shanghai, China), and protein concentrations were determined. Approximately $50 \mu \mathrm{g}$ of protein was separated by $10 \%$ SDS-PAGE, transferred onto a nitrocellulose membrane and incubated with antibodies against TRIP13 (dilution 1:1,000; Abcam, Cambridge, UK). The target proteins were examined with an ECL system (Thermo Fisher Scientific, Inc.) and visualized with X-ray film. Actin (Proteintech Group, Inc., Chicago, IL, USA) was used as the control. Gray value of the western blot bands was performed using ImageJ software (National Institute of Health, Bethesda, MD, USA). The expression intensity of TRIP13 was represented as the ratio of TRIP13 to $\beta$-actin.

Proliferation assay. Cell viability was determined using a CCK-8 assay (Vazyme) according to the manufacturer's instructions. In brief, $24 \mathrm{~h}$ after TRIP13 siRNA transfection, knockdown and control cells were treated with $0.25 \%$ trypsin in EDTA and seeded in clear 96-well plates at a density of 3,000 cells/well. The optical density (OD) of cells was detected at $0,24,48,72$ and $96 \mathrm{~h}$, respectively. CCK-8 reagent $(10 \mu \mathrm{l})$ was added to each well, and cells were incubated at $37^{\circ} \mathrm{C}$ for an additional $2.5 \mathrm{~h}$. Absorbance was measured on a microplate reader at $450 \mathrm{~nm}$ using the medium only wells as blank readings. The experiment was performed eight times with three repeats.

Wound healing assay. Confluent cells from each group (NC and TRIP13-siRNA) were seeded into 6-well plates. A straight scratch was created across the cell layer using a sterile $10-\mu 1$ pipette tip and debris was removed by washing the cells with PBS. Cells were photographed using a Nikon D3300 camera (Nikon Corp., Tokyo, Japan) at 0 and $48 \mathrm{~h}$ following wounding. Assays were repeated three times.

Transwell invasion assay. A Transwell assay was performed to assess the invasion ability of the cells. Transwell invasion plates were pre-coated with Matrigel (BD Biosciences, Franklin Lakes, NJ, USA) and rehydrated using serum-free medium for $4 \mathrm{~h}$ at $37^{\circ} \mathrm{C}$. A total of $600 \mu 1$ medium containing $20 \%$ FBS (Gibco; Thermo Fisher Scientific, Inc.) was added to the lower chamber, while $200 \mu 1$ serum-free medium was added to the upper chamber. At $24 \mathrm{~h}$ after TRIP13 siRNA transfection, knockdown and control cells were harvested using $0.25 \%$ trypsin EDTA and resuspended in serum-free medium to a concentration of $1 \times 10^{6} \mathrm{cells} / \mathrm{ml}$. A total of $200 \mu \mathrm{l}$ cell solution was added to the upper chamber, plates were incubated for $48 \mathrm{~h}$ at $37^{\circ} \mathrm{C}$, and the upper chamber was removed and washed twice in PBS. Cells were then fixed with $4 \%$ paraformaldehyde for $30 \mathrm{~min}$ and stained with $1 \%$ crystal violet (Beyotime Institute of Biotechnology, Haimen, China) for $30 \mathrm{~min}$ at room temperature. Cells in five random fields were counted under an Olympus IX73-FL-PH inverted microscope (Olympus Corp., Tokyo, Japan).

Flow cytometric analysis of cell cycle distribution and apoptosis. For cell cycle analysis, cells were collected, rinsed twice with ice-cold PBS solution and fixed in chilled 70\% ethanol at $-20^{\circ} \mathrm{C}$. The fixed cells were washed with ice-cold
PBS, incubated at room temperature for $20 \mathrm{~min}$ in $1 \mathrm{ml}$ of PBS solution containing $20 \mu \mathrm{g} / \mathrm{ml}$ RNase A and stained with $20 \mu \mathrm{g} / \mathrm{ml}$ of propidium iodide (Sigma-Aldrich) at room temperature for $20 \mathrm{~min}$ before analysis.

For the apoptosis assay, cells were harvested after $48 \mathrm{~h}$ of transfection and stained with an Annexin V-PE/7-AAD Apoptosis Detection kit according to the manufacturer's instructions. Cells were collected, washed with cold PBS and resuspended in $1 \mathrm{X}$ binding buffer at a concentration of $1 \times 10^{3}$ cells $/ \mu 1$. Cells were incubated with $5 \mu \mathrm{l}$ of Annexin V and $5 \mu \mathrm{l}$ of 7-AAD for $15 \mathrm{~min}$ at room temperature in the dark. Binding Buffer (400 $\mu \mathrm{l}$ of 1X) was added and samples were analyzed using flow cytometry. Flow cytometric analysis was performed using FACSCalibur (BD Biosciences). The lower right quadrant in the image represents early apoptosis, while the upper right quadrant represents late apoptosis. The percentage of apoptotic cells reported includes cells in both early and late apoptosis. Data were analyzed with FlowJo software (FlowJo LLC, Ashland, OR, USA).

Statistical analysis. All data are presented as the mean \pm standard deviation. Survival curve was constructed with the Kaplan-Meier method. Significance of TRIP13 expression in association with clinicopathological parameters was calculated using the Chi-square test. A paired t-test was used to analyze the differences of TRIP13 expression levels between HCC tissues and corresponding adjacent normal tissues. The differences among three or more groups in experiments with $\mathrm{HCC}$ cell lines were analyzed by the one-way analysis of variance (ANOVA). The differences between the two groups in experiments of HCC cells were performed with an independent-samples t-test. Statistical calculations were performed using GraphPad Prism software version 6.0 (GraphPad, Inc., La Jolla, CA, USA). P $<0.05$ was considered to indicate a statistically significant difference.

\section{Results}

TRIP13 is upregulated in HCC and high TRIP13 expression predicts poor prognosis. The association between TRIP13 and HCC was evaluated from TCGA data. TCGA data: molecular, clinical and pathological data were downloaded from the TCGA Data Portal (up to Nov 1, 2015) (http://cancergenome. nih.gov/). The results revealed that TRIP13 was overexpressed in the HCC samples $(n=225)$ compared to that noted in the normal liver specimens $(n=220, P<0.0001$, Fig. 1A). Similarly, immunohistochemistry (IHC) revealed that TRIP13 was upregulated in HCC samples compared with that noted in the normal liver tissues (Fig. 1B). The number of TRIP13positive cells (stained nuclei) was counted in 10 randomly chosen high-power-fields (HPF)/section. Quantitative data are shown in Fig. 1C. TCGA data also revealed that TRIP13 expression was significantly elevated in primary HBV or HCV-related HCC tissues compared with HBV or HCV-related normal liver tissues (Fig. 1D). The patients were divided into TRIP13-low and TRIP13-high groups by setting the best performing threshold of gene expression as the cut-off (171.07). The optimal cut-off was defined as the point with the most significant (log-rank test) split. The hazard ratio (HR) with $95 \%$ confidence intervals (CI) and 


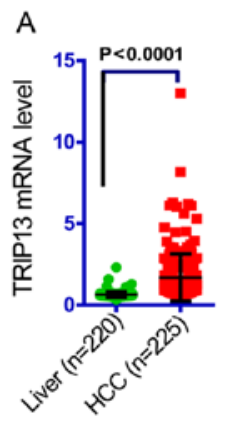

B

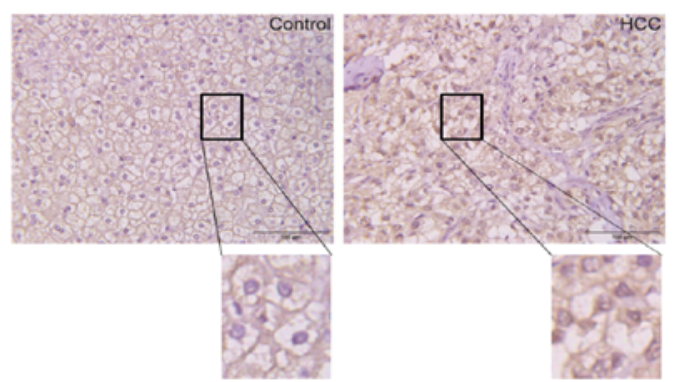

C

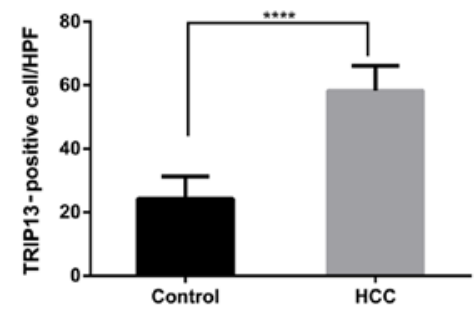

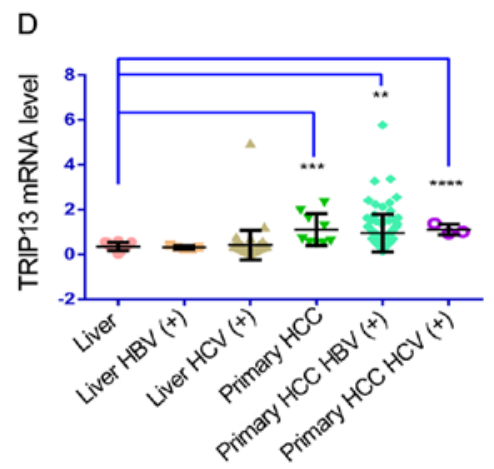

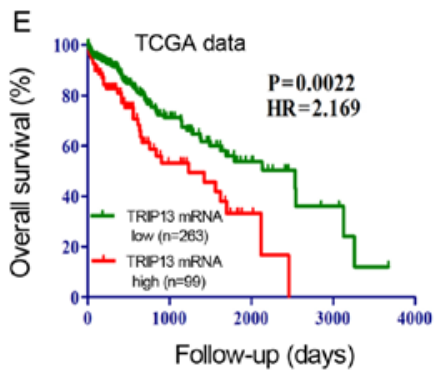

Figure 1. TRIP13 overexpression in hepatocellular carcinoma (HCC). (A) TRIP13 gene expression in The Cancer Genome Atlas (TCGA) database. (B and C) Representative immunohistochemistry (IHC) staining of TRIP13 expression in HCC and matched normal tissues. Scale bar, $100 \mu \mathrm{m}$. (D) TRIP13 expression in HBV or HCV-related HCC tissues and liver tissues from the TCGA database. (E) Overall survival (OS) of patients displaying high or low TRIP13 expression $(\mathrm{P}=0.0022)$. Error bar indicates $\mathrm{SD} .{ }^{* * *} \mathrm{P}<0.01,{ }^{* * *} \mathrm{P}<0.001$ and ${ }^{* * * * *} \mathrm{P}<0.0001$. HPF, high-power field.

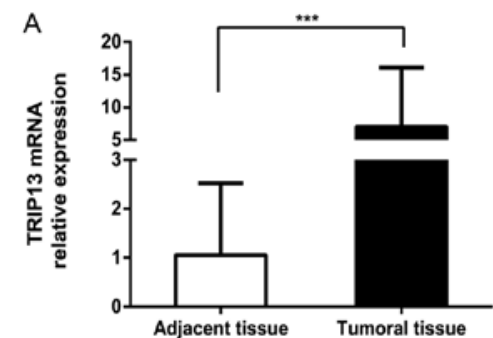

$\circ$

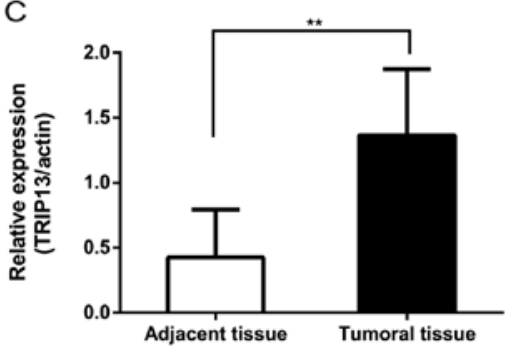

B

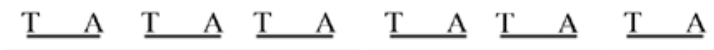

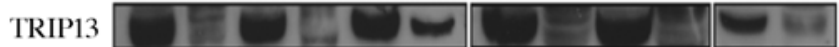
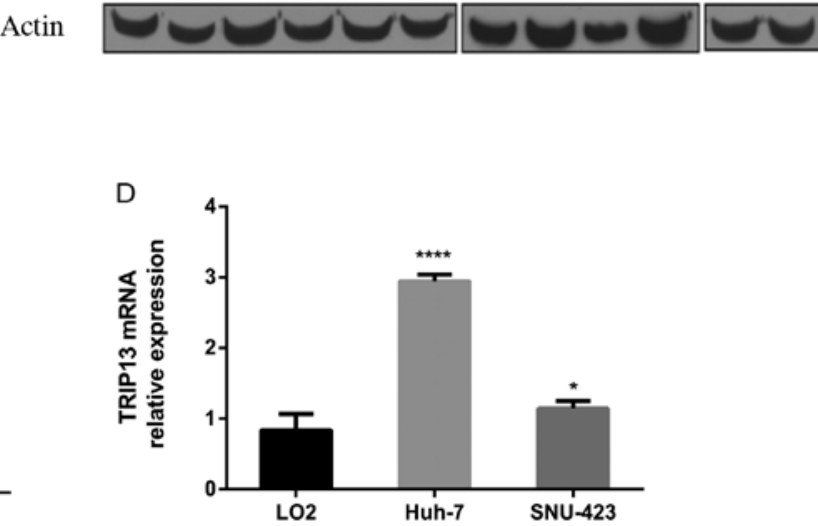

Figure 2. (A) Expression of TRIP13 mRNA in HCC and paired adjacent tissue samples. (B) TRIP13 protein expression in HCC and paired adjacent tissue samples was determined by western blotting. Representative western blots are shown. T, tumoral tissue; A, adjacent tissue. (C) Gray value analysis of western blot experiments normalized against $\beta$-actin. Statistical significance was assessed using a two-tailed Student's t-test. (D) TRIP13 mRNA expression varied among the LO2, Huh-7 and SNU-423 cell lines. Statistical significance was assessed using ANOVA and Tukey's post hoc test. Error bar indicates SD. ${ }^{*}<0.05$, ${ }^{* * *} \mathrm{P}<0.01,{ }^{* * *} \mathrm{P}<0.001$ and ${ }^{* * * * *} \mathrm{P}<0.0001$.

log-rank P-value were calculated. Patients with higher TRIP13 expression had significantly shorter overall survival than patients with lower TRIP13 expression (HR: 2.169, 95\% CI, 1.321-3.559, $\mathrm{P}=0.0022$, Fig. 1E). For better understanding of the clinical relevance of TRIP13 expression in HCC, the relationship between TRIP13 expression and clinicopathological parameters was examined. According to the median value (3.8) of relative TRIP13 expression in HCC tissues, 47 HCC patients were subsequently divided into TRIP13-high $(n=23)$ and TRIP13-low groups $(n=24)$. The statistical analysis revealed that a high level of TRIP13 expression in HCC was significantly correlated with TNM stage $(\mathrm{P}=0.03)$ and tumor size $(\mathrm{P}=0.042)$. However, TRIP13 expression was not associated with the other clinicopathological features such as 
Table II. Association between TRIP13 expression and the clinicopathological characteristics of the 47 HCC patients.

\begin{tabular}{|c|c|c|c|c|}
\hline \multirow[b]{2}{*}{ Characteristics } & \multirow[b]{2}{*}{ Total (n) } & \multicolumn{2}{|c|}{ TRIP13 expression } & \multirow[b]{2}{*}{ P-value } \\
\hline & & Low $(n=24)$ & $\operatorname{High}(\mathrm{n}=23)$ & \\
\hline \multicolumn{5}{|l|}{ Age (years) } \\
\hline$\leq 56$ & 28 & 12 & 16 & 0.238 \\
\hline$>56$ & 19 & 12 & 7 & \\
\hline \multicolumn{5}{|l|}{ Sex } \\
\hline Female & 18 & 6 & 12 & 0.075 \\
\hline Male & 29 & 18 & 11 & \\
\hline \multicolumn{5}{|c|}{ Serum AFP (ng/ml) } \\
\hline$\leq 400$ & 36 & 20 & 16 & 0.318 \\
\hline$>400$ & 11 & 4 & 7 & \\
\hline \multicolumn{5}{|l|}{ HBV infection } \\
\hline Positive & 38 & 18 & 20 & 0.461 \\
\hline Negative & 9 & 6 & 3 & \\
\hline \multicolumn{5}{|c|}{ TNM stage (I/II/III) } \\
\hline $\mathrm{I}+\mathrm{II}$ & 32 & 20 & 12 & 0.03 \\
\hline III & 15 & 4 & 11 & \\
\hline \multicolumn{5}{|l|}{ Tumor size $(\mathrm{cm})$} \\
\hline$\leq 5$ & 24 & 16 & 8 & 0.042 \\
\hline$>5$ & 23 & 8 & 15 & \\
\hline \multicolumn{5}{|c|}{ Lymph node metastasis } \\
\hline Positive & 20 & 9 & 11 & 0.561 \\
\hline Negative & 27 & 15 & 12 & \\
\hline
\end{tabular}

HCC, hepatocellular carcinoma; AFP, $\alpha$-fetoprotein; HBV, hepatitis B virus; TNM, tumor, node, metastasis .
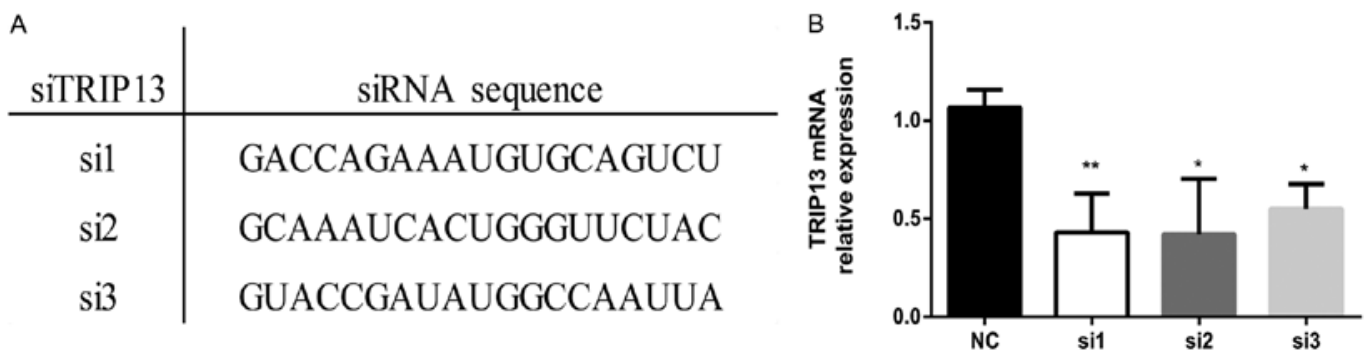

Figure 3. (A) Three siRNAs targeting TRIP13 were (B) analyzed to assess the downregulation of TRIP13. ${ }^{*} \mathrm{P}<0.05,{ }^{* *} \mathrm{P}<0.01$.

age $(\mathrm{P}=0.238)$, sex $(\mathrm{P}=0.075)$, serum level of $\mathrm{AFP}(\mathrm{P}=0.318)$, hepatitis $\mathrm{B}$ virus $(\mathrm{HBV})$ infection $(\mathrm{P}=0.461)$, and lymph node metastasis $(\mathrm{P}=0.561$; Table II). Our data strongly suggest that elevated TRIP13 expression may play a critical role in HCC progression, and may be a valuable biomarker for this disease.

To explore the role of TRIP13 in HCC development, RT-PCR was performed for 47 paired HCC and corresponding adjacent normal tissues. The expression of TRIP13 was consistently elevated in HCC tissues compared to that noted in the paired adjacent normal tissues (Fig. 2A). Western blotting revealed a similar trend (Fig. 2B and C). TRIP13 expression was measured in one human normal liver cell line, LO2, and three HCC cell lines. The results showed that TRIP13 expression was obviously upregulated in Huh-7 and SNU-423 HCC cell lines compared with LO2 (Fig. 2D). Taken together, these data indicate that TRIP13 expression is elevated in HCC and may be involved in its progression.

TRIP13 regulates $H C C$ cell growth and proliferation. To investigate the possible function of TRIP13 in the progression of HCC, the Huh-7 cell line was transfected with special siRNA oligonucleotides against TRIP13 (TRIP13-siRNA group), along with negative control oligonucleotides (NC group). Three siRNAs targeting TRIP13 were analyzed for effectiveness in the downregulation of TRIP13 (Fig. 3A). The efficiency of TRIP13 silencing by siRNAs was evaluated using RT-PCR 


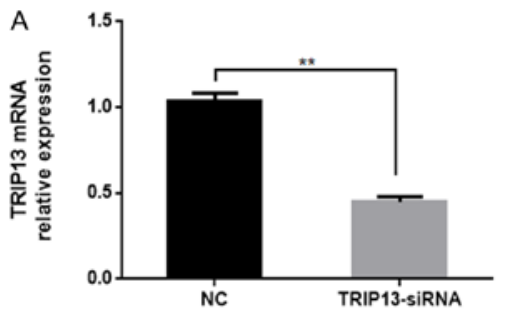

B
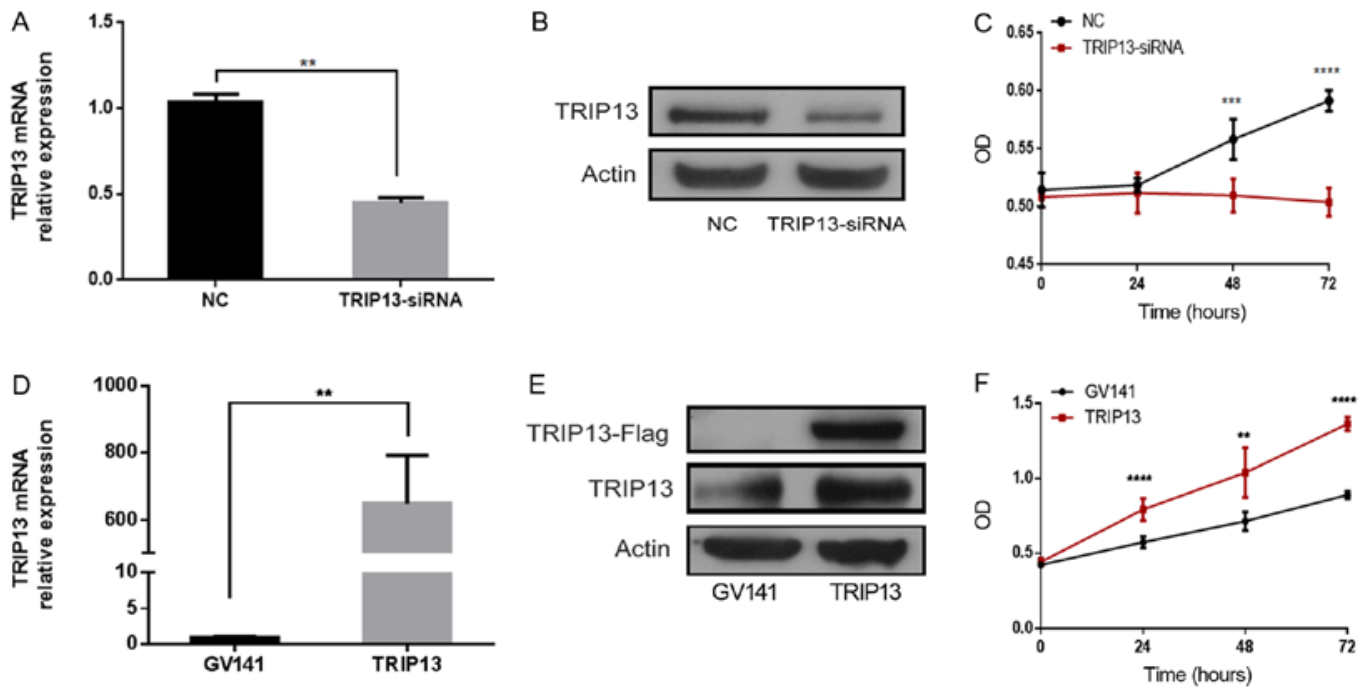

Figure 4. TRIP13 downregulation inhibits Huh-7 cell proliferation. Expression of TRIP13 mRNA (A) and protein (B) in the TRIP13-siRNA and NC groups in Huh-7 cells. (C) Different proliferation abilities of the TRIP13-siRNA and NC groups as measured in Huh-7 cells using a CCK-8 assay. Expression of TRIP13 mRNA (D) and protein (E) in the GV141-empty and GV141-Flag-TRIP13 groups in Huh-7 cells. (F) Different proliferation abilities of the GV141-empty and GV141-Flag-TRIP13 groups as measured by a CCK-8 assay. Statistical significance was assessed using a two-tailed Student's t-test. Error bars indicate SD. ${ }^{* *} \mathrm{P}<0.01,{ }^{* * *} \mathrm{P}<0.001$ and ${ }^{* * * *} \mathrm{P}<0.0001$.

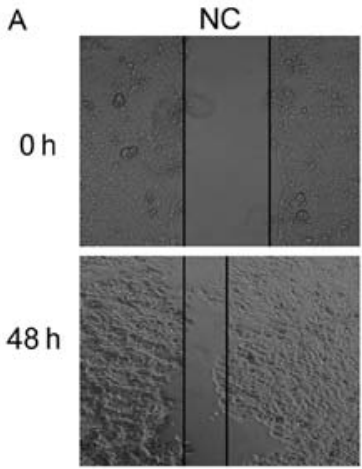

B

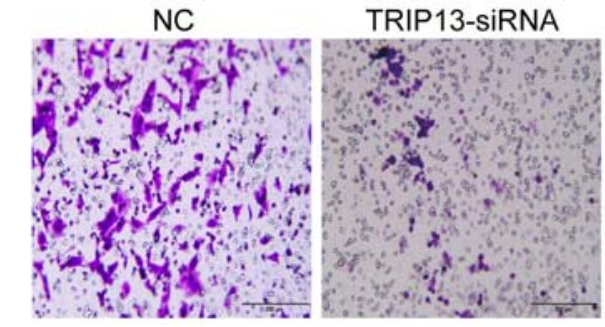

TRIP13-siRNA

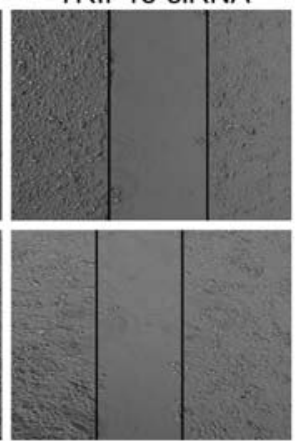

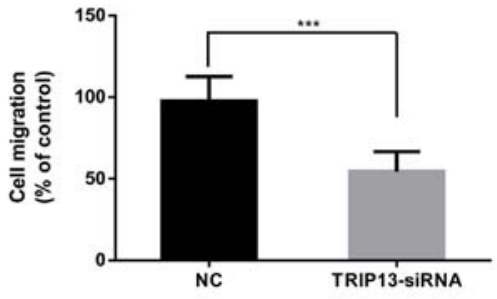

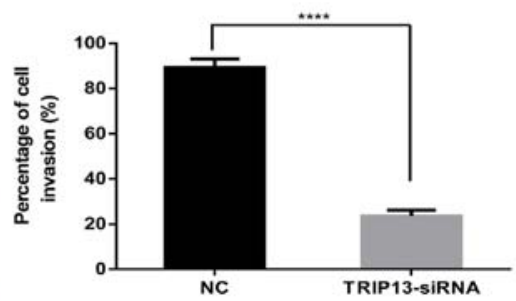

Figure 5. TRIP13 downregulation inhibits Huh-7 cell migration and invasion. (A) The migration of the TRIP13-siRNA and MC groups was measured using a wound healing assay. (B) Decreased invasion was observed in the TRIP13-siRNA group compared with the NC group. Statistical significance was assessed using a two-tailed Student's t-test. Error bar indicates SD. ${ }^{* * *} \mathrm{P}<0.001$ and ${ }^{* * * * *} \mathrm{P}<0.0001$.

in Huh-7 cells (Fig. 3B). The siRNA showing the most sustained knockdown of TRIP13 (si1) was used for subsequent experiments. The result of RT-PCR revealed that TRIP13 mRNA was significantly downregulated in the TRIP13siRNA group compared with the NC group (Fig. 4A). Similar trends were also identified in the protein levels between the two groups (Fig. 4B). The CCK-8 results revealed that, when compared to the NC group, TRIP13 knockout in the TRIP13siRNA group significantly reduced the proliferation of Huh-7 cells following transfection (Fig. 4C). TRIP13 expression was also successfully upregulated in Huh-7 cells via transfection with a plasmid overexpressing TRIP13 (GV141-Flag-TRIP13). Compared with GV141-empty vector transfection, GV141-FlagTRIP13 transfection resulted in increased TRIP13 expression at the mRNA and protein levels (Fig. 4D and E). Huh-7 cells overexpressing TRIP13 exhibited significantly increased cell proliferation compared with the empty vector group (Fig. 4F). Collectively, these results suggest that TRIP13 positively regulates the proliferation of $\mathrm{HCC}$ cells.

TRIP13 downregulation inhibits invasion and migration in HCC cells. To clarify whether TRIP13 functions as a tumor 

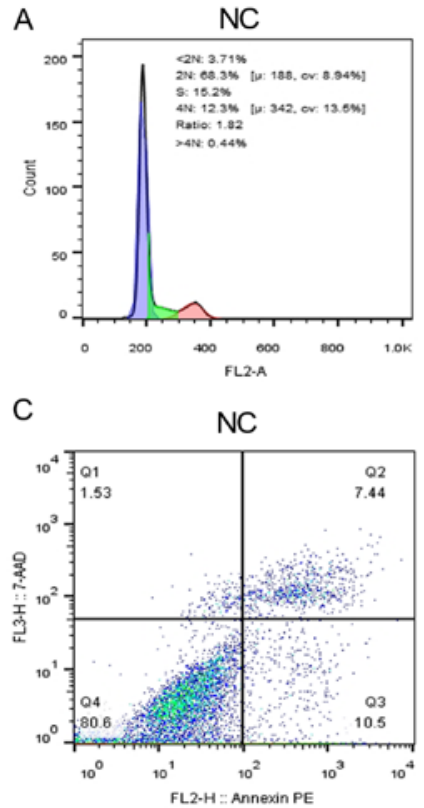
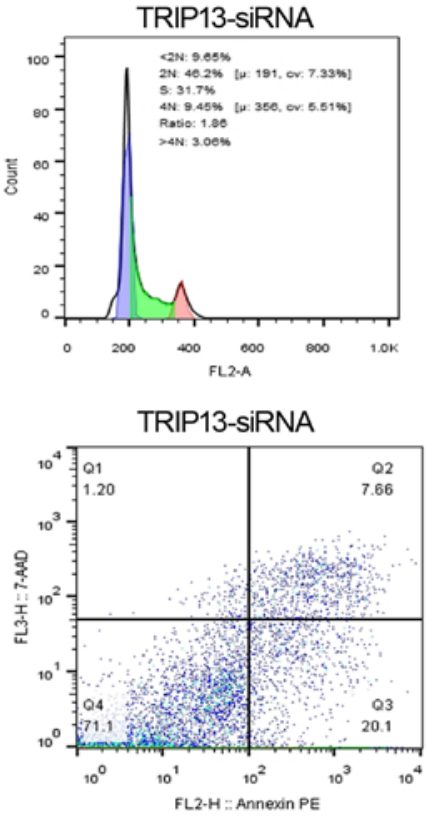

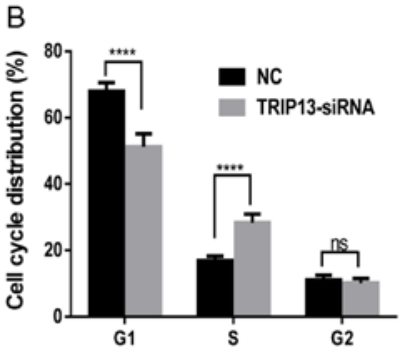

D

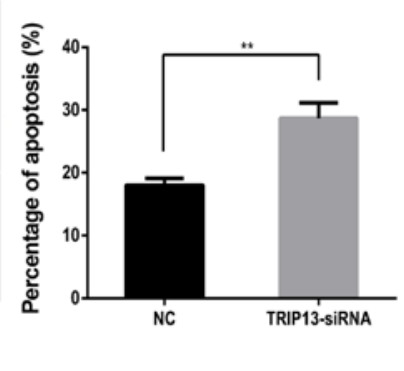

Figure 6. TRIP13 induces Huh-7 cell cycle arrest and apoptosis. (A) The effect of TRIP13 downregulation on the cell cycle distribution of Huh-7 cells. (B) Percentage of cells in each phase. (C) Effect of TRIP13 downregulation on the apoptosis of Huh-7 cells. (D) Percentage of cells undergoing apoptosis as assessed using flow cytometry. Error bar indicate SD. ${ }^{* *} \mathrm{P}<0.01,{ }^{* * * *} \mathrm{P}<0.0001 ;$ ns, not significant.
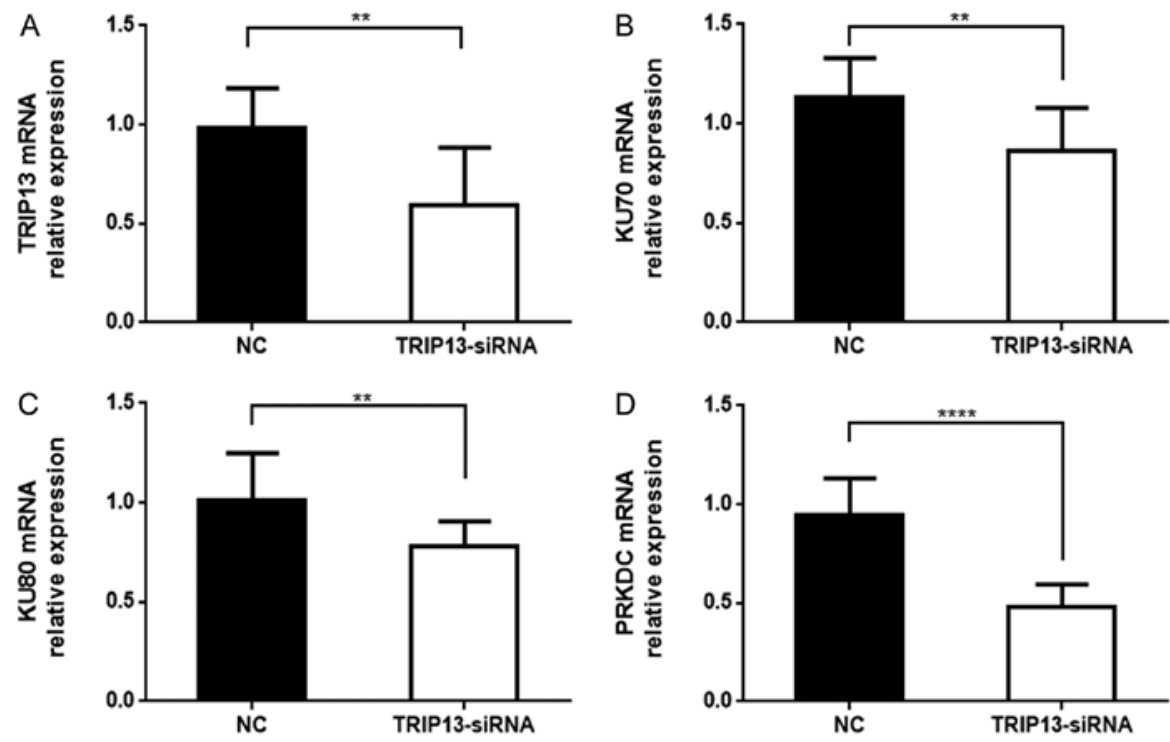

Figure 7. The expression of (A) TRIP13, (B) KU70, (C) KU80 and (D) PRKDC mRNAfollowing transfection with TRIP13-siRNA. Statistical significance was assessed by two-tailed Student's t-test. Error bar indicates SD. ${ }^{* * *} \mathrm{P}<0.01$ and ${ }^{* * * * *} \mathrm{P}<0.0001$.

promoter in the pathogenesis and progression of HCC, we examined the effect of TRIP13 on cell migration and invasion by downregulating TRIP13. The Huh-7 cell line was selected, as the natural TRIP13 expression was high. Wound healing and Transwell invasion assays were performed to explore the possible roles of TRIP13 in migration and invasion. The results of the wound healing and Transwell invasion assays showed that TRIP13 downregulation led to significant decreases in migration (Fig. 5A) and invasion (Fig. 5B) in Huh-7 cells. These findings suggest that TRIP13 may play an important role in the progression of HCC.

TRIP13 downregulation affects cell cycle progression and the rate of apoptosis. To investigate the mechanism by which
TRIP3 promotes proliferation in HCC cells, we investigated whether growth promotion was associated with specific cell cycle control. We first examined cell cycle distribution in Huh-7 cells, using flow cytometry to analyze the DNA content in each cell cycle phase. Compared with the NC-siRNA group, the percentage of cells in the S phase in the TRIP13-siRNA group was significantly increased (Fig. 6A and B). The results indicated that the inhibitory effect of TRIP13 downregulation occurred due to S-phase arrest. Furthermore, we assessed apoptosis in Huh-7 cells with TRIP13 knockdown. We found that when TRIP13 was depleted, cell apoptosis was significantly promoted when compared with the NC cells (Fig. 6C and D). These data suggest that TRIP13 modulation interrupts cell cycle progression and affects cell survival. 
Effect of TRIP13 on NHEJ repair in HCC. NHEJ/DNA repair group proteins include KU70 (XRCC6), KU80 (XRCC5) and DNA-PKcs (PRKDC). Recent studies indicate that TRIP13 binds with KU70 and KU80 to active DNA-PKc for DNA repair, which help cells with DNA damage to escape cell cycle checkpoint and eventual apoptosis. These cells have an increased tendency to become cancer cells. Since KU70, KU80 and DNA-PKcs are members of the NHEJ repair complex, we detected their expression in Huh-7 cells. As shown in Fig. 7, we found that KU70, KU80 and PRKDC expression was reduced in TRIP13-knockdown Huh-7 cells compared with NC cells. The results revealed that TRIP13 knockdown impairs NHEJ repair in $\mathrm{HCC}$.

\section{Discussion}

In the present study, we investigated the functional association between TRIP13 and the development of HCC. We demonstrated that TRIP13 is upregulated in HCC and this was correlated with cancer progression and metastasis. Survival analysis revealed that patients with TRIP13-positive HCC had significantly poorer overall survival compared with patients with TRIP13-negative HCC. TRIP13 knockdown significantly reduced the proliferation of HCC cells. In contrast, TRIP13 overexpression significantly increased cell proliferation. These results indicated that TRIP13 positively regulates the proliferation of HCC cells. Meanwhile, wound healing and Transwell assays revealed that TRIP13 downregulation inhibited migration and invasion in HCC cell lines. We also found that TRIP13 downregulation was able to suppress cell cycle progression by inducing $\mathrm{S}$ cell cycle arrest, as well as inducing cancer cell apoptosis. These results suggest that the activity of TRIP13 is essential for cancer progression.

Elevated TRIP13 expression has been reported to be associated with aggressive progression and poor outcome in multiple cancers $(8,12,17,18)$. Recent studies identified an additional mitosis-regulating role of TRIP13. It has been demonstrated that TRIP13 works in conjunction with the spindle checkpoint silencing protein and Mad2 inhibitor, p31 ${ }^{\text {(comet) }}$, to disassemble the mitotic checkpoint complex (MCC) and promote anaphase $(9,10,14,19)$. TRIP13 overexpression is a hallmark of cancer cells showing chromosomal instability.

TRIP13 is also responsible for efficient DNA repair following ionizing radiation or chemotherapy by promoting NHEJ, enhancing the survival of head and neck tumor cells and causing continued pathological growth (8). NHEJ is one of the most fundamental mechanisms used for sustaining genome stability $(20,21)$. The canonical mechanism of NHEJ in mammalian cells is initiated by the KU70/KU80 heterodimer locating to the broken DNA ends, along with the DNA-dependent protein kinase catalytic subunit (DNA-PKcs) (22-24). DNA-PKcs is a core component of NHEJ and, according to recent findings, a potential chemosensitization target in a number of cancer types $(24,25)$. The present study demonstrated that TRIP13 expression was elevated in HCC, while TRIP13 knockdown impaired NHEJ repair in $\mathrm{HCC}$ by downregulating the expression of KU70, KU80 and DNA-PKcs (PRKDC). TRIP13 overexpression may promote chromosomal instability in cancer cells, resulting in more malignant characteristics. Developing a greater understanding of the oncogenic mechanisms of TRIP13 in the progression of HCC may be beneficial for improving future treatments.

In conclusion, TRIP13 is an independent prognostic factor for OS that is upregulated in HCC. Decreasing the expression of TRIP13 may effectively inhibit the proliferation, migration and invasion of HCC cells. TRIP13 downregulation exerts an inhibitory effect on HCC cells by arresting the cell cycle in $\mathrm{S}$ phase and inducing apoptosis. These results suggest that suppression of the TRIP13 gene may be an effective therapeutic strategy for HCC treatment.

\section{Acknowledgements}

Not applicable.

\section{Funding}

The present study was supported by Nantong Science and Technology Bureau (grant nos. MS22015105 and MS3201605), the Natural Science Foundation of Jiangsu Province (grant no. BK20160420), the National Natural Science Foundation of China (grant no. 81600449), the National Natural Science Foundation of Young Scientists of China (grant no. 81602443), and the Health Bureau of Nantong City (grant no. WQ2016011).

\section{Availability of data and materials}

All the data in this study were obtained from The Cancer Genome Atlas dataset (TCGA; http://cancergenome.nih.gov/).

\section{Authors' contributions}

LJ and XL acquired the data and created a draft of the manuscript; LJ, JS and RL prepared the experimental materials, performed the statistical analysis and analyzed the results; LJ, YW and ZB wrote, revised and approved the final version of the manuscript. YW and ZB were also involved in the conception and design of the study. All authors read and approved the manuscript and agree to be accountable for all aspects of the research in ensuring that the accuracy or integrity of any part of the work are appropriately investigated and resolved.

\section{Ethics approval and consent to participate}

The study was approved by the Ethics Committee of Jiangsu Province Medical Association and written informed consent was obtained from all patients before tissue samples were harvested.

\section{Patient consent for publication}

Not applicable.

\section{Competing interests}

The authors declare that they have no competing interests. 


\section{References}

1. Cui X, Li Z, Gao J, Gao PJ, Ni YB and Zhu JY: Elevated CXCL1 increases hepatocellular carcinoma aggressiveness and is inhibited by miRNA-200a. Oncotarget 7: 65052-65066, 2016.

2. Zhou B, Chen H, Wei D, Kuang Y, Zhao X, Li G, Xie J andChen P: A novel miR-219-SMC4-JAK2/Stat3 regulatory pathway in human hepatocellular carcinoma. J Exp Clin Cancer Res 33: 55, 2014.

3. Li J, Qiu X, Guo W, Yan B and Zhang S: Prospective analysis of tiopronin in prevention of sorafenib and antiviral therapy inducing liver toxicity in advanced hepatitis B virus-related hepatocellular carcinoma. Med Oncol 32: 238, 2015.

4. Chen J, Rajasekaran M, Xia H, Zhang X, Kong SN, Sekar K, Seshachalam VP, Deivasigamani A, Goh BK, Ooi LL, et al: The microtubule-associated protein $\mathrm{PRCl}$ promotes early recurrence of hepatocellular carcinoma in association with the $\mathrm{Wnt} / \beta$ catenin signalling pathway. Gut 65: 1522-1534, 2016.

5. Shirvani-Dastgerdi E, Schwartz RE and Ploss A: Hepatocarcinogenesis associated with hepatitis $\mathrm{B}$, delta and $\mathrm{C}$ viruses. Curr Opin Virol 20: 1-10, 2016.

6. Tsai CL, Hsu FM and Cheng JC: How to improve therapeutic ratio in radiotherapy of HCC. Liver Cancer 5: 210-220, 2016.

7. Trojan J, Zangos S and Schnitzbauer AA: Diagnostics and treatment of hepatocellular carcinoma in 2016: Standards and developments. Visc Med 32: 116-120, 2016.

8. Banerjee R, Russo N, Liu M, Basrur V, Bellile E, Palanisamy N, Scanlon CS, Van Tubergen E, Inglehart RC, Metwally T, et al: TRIP13 promotes error-prone nonhomologous end joining and induces chemoresistance in head and neck cancer. Nat Commun 5: 4527, 2014

9. Ye Q, Rosenberg SC, Moeller A, Speir JA, Su TY and Corbett KD: TRIP13 is a protein-remodeling AAA+ ATPase that catalyzes MAD2 conformation switching. Elife 4: 4, 2015.

10. Ma HT and Poon RYC: TRIP13 regulates both the activation and inactivation of the spindle-assembly checkpoint. Cell Reports 14: 1086-1099, 2016

11. Tipton AR, Wang K, Oladimeji P, Sufi S, Gu Z and Liu ST: Identification of novel mitosis regulators through data mining with human centromere/kinetochore proteins as group queries BMC Cell Biol 13: 15, 2012.

12. Tao Y, Yang G, Yang H, Song D, Hu L, Xie B, Wang H, Gao L, Gao M, Xu H, et al: TRIP13 impairs mitotic checkpoint surveillance and is associated with poor prognosis in multiple myeloma. Oncotarget 8: 26718-26731, 2017.
13. Wang K, Sturt-Gillespie B, Hittle JC, Macdonald D, Chan GK, Yen TJ and Liu ST: Thyroid hormone receptor interacting protein 13 (TRIP13) AAA-ATPase is a novel mitotic checkpoint-silencing protein. J Biol Chem 289: 23928-23937, 2014.

14. Nelson CR, Hwang T, Chen PH and Bhalla N: TRIP13 $3^{\mathrm{PCH}-2}$ promotes Mad2 localization to unattached kinetochores in the spindle checkpoint response. J Cell Biol 211: 503-516, 2015.

15. Van Kester MS, Borg MK, Zoutman WH, Out-Luiting JJ, Jansen PM, Dreef EJ, Vermeer MH, van Doorn R, Willemze R and Tensen CP: A meta-analysis of gene expression data identifies a molecular signature characteristic for tumor-stage mycosis fungoides. J Invest Dermatol 132: 2050-2059, 2012.

16. Livak KJ and Schmittgen TD: Analysis of relative gene expression data using real-time quantitative PCR and the $2^{-\Delta \Delta \mathrm{C}_{\mathrm{T}}}$ method. Methods 25: 402-408, 2001.

17. Zhou K, Zhang W, Zhang Q, Gui R, Zhao H, Chai X, Li Y, Wei $\mathrm{X}$ and Song Y: Loss of thyroid hormone receptor interactor 13 inhibits cell proliferation and survival in human chronic lymphocytic leukemia. Oncotarget 8: 25469-25481, 2017.

18. Kurita K, Maeda M, Mansour MA, Kokuryo T, Uehara K, Yokoyama Y, Nagino M, Hamaguchi M and Senga T: TRIP13 is expressed in colorectal cancer and promotes cancer cell invasion. Oncol Lett 12: 5240-5246, 2016.

19. Miniowitz-Shemtov S, Eytan E, Kaisari S, Sitry-Shevah D and Hershko A: Mode of interaction of TRIP13 AAA-ATPase with the Mad2-binding protein p31comet and with mitotic checkpoint complexes. Proc Natl Acad Sci USA 112: 11536-11540, 2015.

20. Reid DA and Rothenberg E: Repair of chromosomal breaks by NHEJ. Oncotarget 6: 15730-15731, 2015.

21. Hentges P, Waller H, Reis CC, Ferreira MG and Doherty AJ: Cdk1 restrains NHEJ through phosphorylation of XRCC4-like factor Xlf1. Cell Reports 9: 2011-2017, 2014.

22. Zhang LY, Chen LS, Sun R, Ji SJ, Ding YY, Wu J and Tian Y: Effects of expression level of DNA repair-related genes involved in the NHEJ pathway on radiation-induced cognitive impairment. J Radiat Res 54: 235-242, 2013.

23. Bartlett EJ, Brissett NC, Plocinski P, Carlberg T and Doherty AJ: Molecular basis for DNA strand displacement by NHEJ repair polymerases. Nucleic Acids Res 44: 2173-2186, 2016.

24. Newman EA, Lu F, Bashllari D, Wang L, Opipari AW and Castle VP: Alternative NHEJ pathway components are therapeutic targets in high-risk neuroblastoma. Mol Cancer Res 13: 470-482, 2015.

25. Pascale RM, Joseph C, Latte G, Evert M, Feo F and Calvisi DF: DNA-PKcs: A promising therapeutic target in human hepatocellular carcinoma? DNA Repair 47: 12-20, 2016. 